\title{
Some New Intuitionistic Equivalents of Zorn's Lemma
}

\author{
John L. Bell
}

In classical set theory, Zorn's Lemma is equivalent to the axiom of choice and a host of other principles and theorems. But in intuitionistic set theory (IZF), in which the law of excluded middle is not assumed, the situation is quite different. (A presentation of IZF may be found in Chapter VIII of [3].) Here, Zorn's lemma turns out to be remarkably weak: not only does it fail to imply the axiom of choice, but one cannot even prove from it, for example, the Boolean prime ideal theorem or the Stone representation theorem. (This is because, as shown in [4], Zorn's lemma has no nonconstructive purely logical consequences, while both the axiom of choice and the Stone representation theorem imply the law of excluded middle, and the Boolean prime ideal theorem implies the nonconstructive form of de Morgan's law: see [5].) In fact, the vast majority of the assertions intuitionistically provable from Zorn's lemma make explicit mention of the notion of maximality: for example, the existence of maximal chains in partially ordered sets and the maximal ideal theorem for rings. (A conspicuous exception to this is the Sikorski extension theorem for complete Boolean algebras which is intuitionistically derivable from Zorn's lemma: see [4].) In this note two apparently new results are proved, neither of which make explicit reference to maximality - the one a fixpoint theorem for complete lattices, the other a result concerning binary relations-and each is shown to be intuitionistically equivalent to Zorn's lemma.

We begin with some 


\section{Definitions.}

Let $P$ be a partially ordered set. An element $a$ of $P$ is maximal if, for any $x \in P, a \leq x$ implies $a=x . P$ is inductive if each chain $X$ in $P$ has a supremum or join $\bigvee X$. By Zorn's Lemma is meant the assertion that any inductive partially ordered set with an element has a maximal element.

A subset $B$ of $P$ is a base for $P$ if, for any $x, y \in P$, we have

$$
\forall b \in B[b \leq x \Rightarrow b \leq y] \Rightarrow x \leq y .
$$

Notice that if $P$ is a complete lattice, $B$ is a base iff

$$
\forall x \in L . x=\bigvee\{b \in B: b \leq x\} .
$$

A map $f: P \rightarrow P$ is (i) self-adjoint if for any $x, y \in P$ we have

$$
x \leq f(y) \Leftrightarrow y \leq f(x),
$$

and (ii) inflationary on a subset $X \subseteq P$ if $x \leq f(x)$ for all $x \in X$.

Lemma. Let $P$ be a partially ordered set and $f: P \rightarrow P$ a self-adjoint map. Let $X$ be a subset of $P$ with a supremum $\bigvee X$. Then $f[X]$ has infimum $\Lambda f X]=f(\bigvee X)$.

Proof. We have, for any $y \in P$

$$
\forall x \in X . y \leq f(x) \Leftrightarrow \forall x \in X . x \leq f(y) \Leftrightarrow \bigvee X \leq f(y) \Leftrightarrow y \leq f(\bigvee X) .
$$

It follows in particular that any self-adjoint map on a partially ordered set is order-inverting.

We use this to establish what we shall term the

Fixpoint Property (for self-adjoint maps). Assume Zorn's Lemma. Let $f: L \rightarrow L$ be a self-adjoint map on a complete lattice $L$ possessing a base $B$ on which $f$ is inflationary. Then $f$ has a fixpoint.

Proof. Let $D=\{x \in L: x \leq f(x)\}$. We claim that, with the order inherited from $L, D$ is inductive. For consider any chain $C$ in $D$, and let $c=\bigvee C$. We claim that $c \in D$. To prove this, we note that $f(c)=f(\bigvee C)=$ 
$\Lambda f[C]$ by the lemma, so it suffices to show that $c \leq \Lambda f[C]$, i.e. $x \leq f(y)$ for all $x, y \in C$. Now if $x, y \in C$, then either $x \leq y$ or $y \leq x$. In the first case $x \leq y \leq$ $f(y)$; in the second $f(x) \leq f(y)$ so that $x \leq f(x) \leq f(y)$.

Accordingly $D$ is inductive and so by Zorn's Lemma has a maximal element $m$. We claim that $f(m)=m$. To prove this it suffices to show that $f(m) \leq m$; since $B$ is a base, for this it suffices in turn to prove that

$$
\forall b \in B[b \leq f(m) \Rightarrow b \leq m] .
$$

Since $m$ is maximal in $D$, to prove $\left(^{*}\right)$ it clearly suffices to prove

$$
\forall b \in B[b \leq f(m) \Rightarrow m \vee b \in D],
$$

i.e.

$$
\forall b \in B[b \leq f(m) \Rightarrow m \vee b \leq f(m \vee b)],
$$

i.e.

$$
\forall b \in B[b \leq f(m) \Rightarrow m \vee b \leq f(m) \wedge f(b)]
$$

So suppose $b \in B$ and $b \leq f(m)$. We already know that $m \leq f(m)$, and $m \leq f(b)$ follows from $b \leq f(m)$ and the self-adjointness of $f$. Thus $m \leq f(m) \wedge f(b)$. Also $b \leq f(m) \wedge f(b)$ since we are given $b \leq f(m)$ and $f$ is inflationary on $B$. Hence $m \vee b \leq f(m) \wedge f(b)$ as required, and $\left(^{* *}\right)$ follows.

The Fixpoint Theorem is proved.

We next make the

Definition. Let $R$ be a binary relation on a set $A$. An $R$-clique in $A$ is a subset $U$ of $A$ such that

$$
\forall x \in A[x \in U \Leftrightarrow \forall y \in U . x R y]
$$

The Clique Property is the assertion that, for any reflexive symmetric binary relation $R$, an $R$-clique exists. 
Finally we prove the

Theorem. The following are equivalent in intuitionistic set theory:

1. Zorn's Lemma (ZL)

2. The Fixpoint Property (FP)

3. The Clique Property (CP).

Proof.

$\mathbf{Z L} \Rightarrow \mathbf{F P}$ has been established above.

$\mathbf{F P} \Rightarrow \mathbf{C P}$. Let $R$ be a symmetric reflexive binary relation on a set $A$. Define the function $F$ on the power set $\operatorname{Pow}(A)$ of $A$ to itself by

$$
F(X)=\{y \in A: \forall x \in X . x R y\} .
$$

The symmetry of $R$ is tantamount to the self-adjointness of $F$ and the reflexivity of $R$ to the assertion that $F$ is inflationary on the base $\{\{a\}: a \in A\}$ for $\operatorname{Pow}(A)$. Accordingly FP yields a fixpoint $U \in P A$ for $F$, that is, an $R$-clique in $A$.

$\mathbf{C P} \Rightarrow \mathbf{Z L}$. Let $(P, \leq)$ be an inductive partially ordered set, and define $R$ to be the symmetric reflexive relation $x \leq y \vee y \leq x$ on $P$. CP yields an $R$ clique $U$ in $P$, which is evidently a chain in $P$, and so, by the inductivity of $P$, has an upper bound $u$. We claim that $u$ is a maximal element of $P$. For suppose $u \leq x$. Then clearly $\forall y \in U$. $x R y$, whence $x \in U$, and so $x \leq u$. Therefore $x=u$, and $u$ is maximal.

Remarks. 1. The equivalence between FP and $\mathbf{C P}$ may be further explicated by the following observation. Let $f$ be a self-adjoint map on a complete lattice $L$ which is inflationary on a set $B$ of generators, and let $R$ be the symmetric reflexive relation $x \leq f(y)$ on $B$. Then there are mutually inverse correspondences $\varphi, \psi$ between the set $F$ of fixpoints of $f$ (which is easily shown to coincide with the set of maximal elements of $\{x \in L: x \leq f(x)\})$ and the set $\mathscr{C}$ of $R$-cliques. These correspondences are 
given, respectively, by $\varphi(m)=\{x \in B: x \leq m\}$ for $m \in F$ and $\psi(X)=\bigvee X$ for $X$ $\in \mathscr{C}$.

2. The relationship just described can be generalized to an adjunction between categories. Let $\mathscr{R} e l$ be the category whose objects are pairs $(A, R)$ with $R$ a reflexive symmetric reflexive relation on a set $A$, and with relation-preserving maps as arrows. Let Lat be the category whose objects are triples $(L, B, f)$ with $L$ a complete lattice, $B$ a subset of $L$, and $f$ a self-adjoint map on $L$ which is inflationary on $B$; an arrow $p:(L, B, f) \rightarrow$ $\left(L^{\prime}, B^{\prime}, f\right)$ in $\mathscr{L} a t$ is a $\bigvee$-preserving map $L \rightarrow L^{\prime}$ sending $B$ into $B^{\prime}$ such that $p(f(x)) \leq f^{\prime}(p(x))$ for all $x \in L$. We define the functors $F$ : Rel $\rightarrow$ Lal and $G:$ Lat $\rightarrow$ Rel as follows. Given $\mathbf{A}=(A, R)$ and $h: \mathbf{A} \rightarrow\left(A^{\prime}, R^{\prime}\right)=$ $\mathbf{A}^{\prime}$ in $\mathscr{R} e l$, we define $F \mathbf{A}=(\operatorname{Pow}(A),\{\{a\}: a \in A\}, \tilde{R})$ with $\tilde{R}(X)=\{y \in A: \forall x \in X . x R y\} ;$ and $F h: F \mathbf{A} \rightarrow F \mathbf{A}^{\prime}$ by $(F h)(X)=\{h(x): x \in X\}$. Given $\mathbf{L}=(L, B, f)$ and $p: \mathbf{L} \rightarrow\left(L^{\prime}, B^{\prime}, f\right)$ in Lat we define $G \mathbf{L}=\left(B, f^{*}\right)$, where $f^{*}$ is defined by $x f^{*} y$ iff $x \leq f(y)$ and $G p$ is the restriction of $p$ to $B$.

Then $F$ is left adjoint to $G$, and the unit of the adjunction is iso. So $F$ is full and faithful, and thus $\mathscr{R} e$ is, up to isomorphism, a full coreflective subcategory of $\mathscr{L} a t$. The objects $\mathbf{L}=(L, B, f)$ of $\mathscr{L}$ at for which the counit arrow $F G \mathbf{L} \rightarrow \mathbf{L}$ is epi are precisely those in which $B$ is a base for $L$ : call such objects based. The adjunction $F \dashv G$ then restricts to one between $\mathscr{R} e l$ and $\mathscr{L a t}$ 's full subcategory $\mathscr{L} a t^{*}$ of based objects. So $\mathscr{R} e l$ is also, up to isomorphism, a full coreflective subcategory of $\mathscr{L a l}^{*}$.

3. Our discussion has been conducted within IZF, in which, although no use of the law of excluded middle is made, the power set axiom is assumed. The question arises as to what extent the arguments 
formulated here can be carried over to constructive set theory CZF, the "predicative" version of intuitionistic set theory in which the power set axiom is not assumed. (A presentation of CZF may be found in [1].) In [2], Peter Aczel has shown that, in CZF, Zorn's Lemma (suitably formulated) continues to imply FP and $\mathbf{C P}$, and that these latter remain equivalent there. But, in contrast with the situation in IZF, they do not appear to yield Zorn's Lemma in CZF. The claims in Remarks 1 and 2, however, carry over to CZF.

Acknowledgments. I am grateful to Peter Aczel for his illuminating observations on the contents of this paper, and to Andreas Blass for his helpful suggestions for improving it.

\section{References}

[1] Aczel, P. and Rathjen, M. Notes on Constructive Set Theory. Report No. 40, 2000/2001, Institut Mittag-Leffler, Royal Swedish Academy of Sciences.

[2] Aczel, P. Zorn's Lemma in CZF. Preprint, Aug. 17, 2002.

[3] Beeson, M. J. Foundations of Constructive Mathematics. SpringerVerlag, 1980.

[4] Bell, John L. Zorn's Lemma and Complete Boolean Algebras in Intuitionistic Type Theories. Journal of Symbolic Logic 62 (1997), 4, 1265-1279.

[5] Bell, John L. Boolean Algebras and Distributive Lattices Treated Constructively. Mathematical Logic Quarterly 45 (1999), 1, 135-143. 